\title{
Influence of the foreshock of the Earth's bow shock on the interplanetary shock propagation during their mutual interaction
}

\author{
Lubomír Přech, Zdeněk Němeček, and Jana Šafránková \\ Charles University, Faculty of Mathematics and Physics, V Holesovickach 2, 18000 Praha 8, Czech Republic
}

(Received October 27, 2007; Revised April 13, 2008; Accepted April 14, 2008; Online published May 29, 2009)

\begin{abstract}
Interplanetary shocks have been recognized as a very efficient source of geomagnetic disturbances. We present a short study of the propagation of one interplanetary (IP) shock observed by five spacecraft located in the solar wind far upstream of the Earth's bow shock as well as in its close vicinity. The IP shock normal was highly inclined from the Sun-Earth line and thus the IP shock-bow shock interaction started at the flank. We have found a significant evolution of IP shock parameters during its motion along the bow shock. This modification is discussed and attributed to the presence of strong fluxes of energetic particles in the foreshock.
\end{abstract}

Key words: Solar wind-magnetosphere interaction, upstream conditions, interplanetary shock, foreshock.

\section{Introduction}

Geospace is affected by the solar wind, a supersonic plasma stream emerging from the Sun. Besides large-scale structures (such as the interplanetary magnetic field sector and solar wind flow regime boundaries), eruptive solar events of high intensity, predominantly solar flares resulting in halo Coronal Mass Ejections (CMEs) and solar energetic particle emissions, can have a significant impact on geospace. The majority of the very intense storms were observed to be associated with interplanetary CMEs (ICMEs) and shocks passing by the Earth (Tsurutani and Gonzalez, 1997). They are an interplanetary manifestation of earthward directed CMEs. For example, interplanetary pressure events, like interplanetary (IP) shocks, compress or expand the magnetosphere and increase or decrease the magnetopause and tail currents that result in changes of other near-Earth current systems (Le et al., 1998). The geoeffectiveness of the solar wind structures and discontinuities is related to their 3D geometry and orientation. Since multispacecraft observations are necessary for the determination of the 3D geometry and structure of shocks (and/or other structures in the solar wind) (e.g., Thomsen, 1988), not too many experimental studies have attempted to address this problem.

Several studies have been done in the recent years analyzing the solar wind interplanetary magnetic structures that can be geoeffective (e.g., review by Gonzalez et al., 1999). Vennerstroem (2001) examined 30 years of satellite measurements of the solar wind during magnetic storms and he had estimated the relative importance of the ejection of magnetic structures from the Sun and the stream interaction processes during solar wind propagation in generating intense southward magnetic fields in the interplan-

Copyright (c) The Society of Geomagnetism and Earth, Planetary and Space Sciences (SGEPSS); The Seismological Society of Japan; The Volcanological Society of Japan; The Geodetic Society of Japan; The Japanese Society for Planetary Sciences; TERRAPUB etary medium. Echer et al. (2006) presented a statistical study of the geoeffectiveness of the solar wind magnetic interplanetary structures including magnetic clouds (MCs), corotating interaction regions (CIRs) and interplanetary (IP) shocks over a wide observational period. They observed that magnetic clouds are more efficient than shocks or CIRs in producing all geomagnetic disturbances and they have confirmed that compound structures (shocks and MCs) are more geoeffective in every type of magnetospheric activity than isolated structures. However, the application of these findings in the space weather models requires a precise forecasting of the structure arrival to the Earth that is generally a difficult tasks (see e.g., Fry et al., 2003 or McKenna-Lawlor et al., 2006).

A strong correlation between IP shocks impinging on the magnetosphere and geomagnetic disturbances has been reported by many authors (e.g., Gonzalez et al., 1999). The interaction of IP shocks with the Earth's bow shock and their transmissions through the magnetosheath to the boundary of the magnetosphere has been studied mainly by a gas dynamic modeling (e.g., Dryer, 1973; Grib et al., 1979; Spreiter and Stahara, 1994). Generally, it is assumed that the incoming IP shocks are planar on the scale-size of the magnetosphere in the undisturbed solar wind. Russell et al. (2000) analyzed a single IP shock with four solar wind spacecraft and found that normals calculated from the data of three of them were consistent with the planarity assumption (with the accuracy of the travel time estimates). On the other hand, a deviation from planarity has also been reported (e.g., Russell et al., 1983; Safrankova et al., 1998).

Two papers by Koval et al. $(2005,2006)$ demonstrates that the shock front in the magnetosheath is inclined and this inclination results in a delay of the shock arrival to the magnetopause. A non-planar shock propagation through the magnetosheath resulting from experimental observations has been confirmed by two numerical MHD simulation results (Koval et al., 2006). 
The short survey has shown that the problem of the interaction of an IP shock with the Earth's magnetosphere is not fully explained. Although MHD modeling achieved a particular success in description of the IP shock interaction with the bow shock (e.g., Zhuang et al., 1981; Grib, 1982; Yan and Lee, 1996; Samsonov et al., 2006), the models have two significant limitations: (1) their present codes can be applied only to the shocks perpendicular to the solar wind velocity and (2) they cannot account for foreshock effects.

In the paper, we have identified one IP shock that was registered by 5 spacecraft in the solar wind. Two of them were located far upstream and three others in a close vicinity of the bow shock in different positions with respect to the expected foreshock boundary. Moreover, the shock normal determined either by the 4-spacecraft method or from Rankine-Hugoniot relations (this method solves the full set of Rankine-Hugoniot equations according to the Vinas and Scudder (1986) paper) was declined on a large angle from the Sun-Earth line. The paper is devoted to a comprehensive study of this event and it is oriented to a preliminary analysis of the foreshock effects.

\section{Observation}

The IP shock (probably CIR-driven) was first observed by ACE near the L1 point at 0007 UT on August 10, 1998 and about 24 minutes later by WIND at the distance about $80 R_{\mathrm{E}}$ from the Earth. As can be seen in Fig. 1, both spacecraft observed nearly rectangular jumps of all parameters; the solar wind speed changed from $\sim 410$ to $\sim 440 \mathrm{~km} / \mathrm{s}$ and the density from $\sim 4$ to $\sim 9 \mathrm{~cm}^{-3}$. These jumps suggest a rather weak IP shock but the shock parameters calculated from the WIND magnetic field and plasma data using Rankine-Hugoniot relations show that its Alfvénic Mach number is as high as $6.6\left(v_{\mathrm{A}}=54 \mathrm{~km} / \mathrm{s}\right)$ in the normal incidence frame. The reason is that, although the shock speed is only $355 \mathrm{~km} / \mathrm{s}$ in the observer reference frame, it is largely (on $\sim 50^{\circ}$ ) declined from the Sun-Earth line because the shock normal vector is $n=[-0.53 ;-0.44 ; 0.73]$ and the shock speed in the solar wind frame is $346 \mathrm{~km} / \mathrm{s}$. The shock can be classified as quasiperpendicular, $\Theta_{\mathrm{BN}}=\sim 63^{\circ}$.

The same IP shock was observed later by IMP 8 , Geotail, and Interball-1 in front of the bow shock. Since their locations are very important for the present study, we are bringing a sketch in Fig. 2. The spacecraft positions were rotated into one plane but it should be noted that all of them were very close to the ecliptic plane. We have used the timing of the shock observations by ACE, WIND, IMP 8, and GEOTAIL and estimated the shock parameters. These parameters are given in the captions of Fig. 2 and one can note that they are very similar to those derived from WIND measurements. This fact confirms that the shock can be considered to be planar on the scale of the spacecraft separation. The cross-section of the shock plane with the $X Y_{\mathrm{GSE}}$ plane is shown as a dashed line in the figure.

Taking into account the shock orientation, one would expect that the shock would arrive nearly simultaneously to IMP 8 and Geotail and with a significant delay to Interball1. We have chosen the magnetic field strength and ion flux for a comparison of observation in different points that is

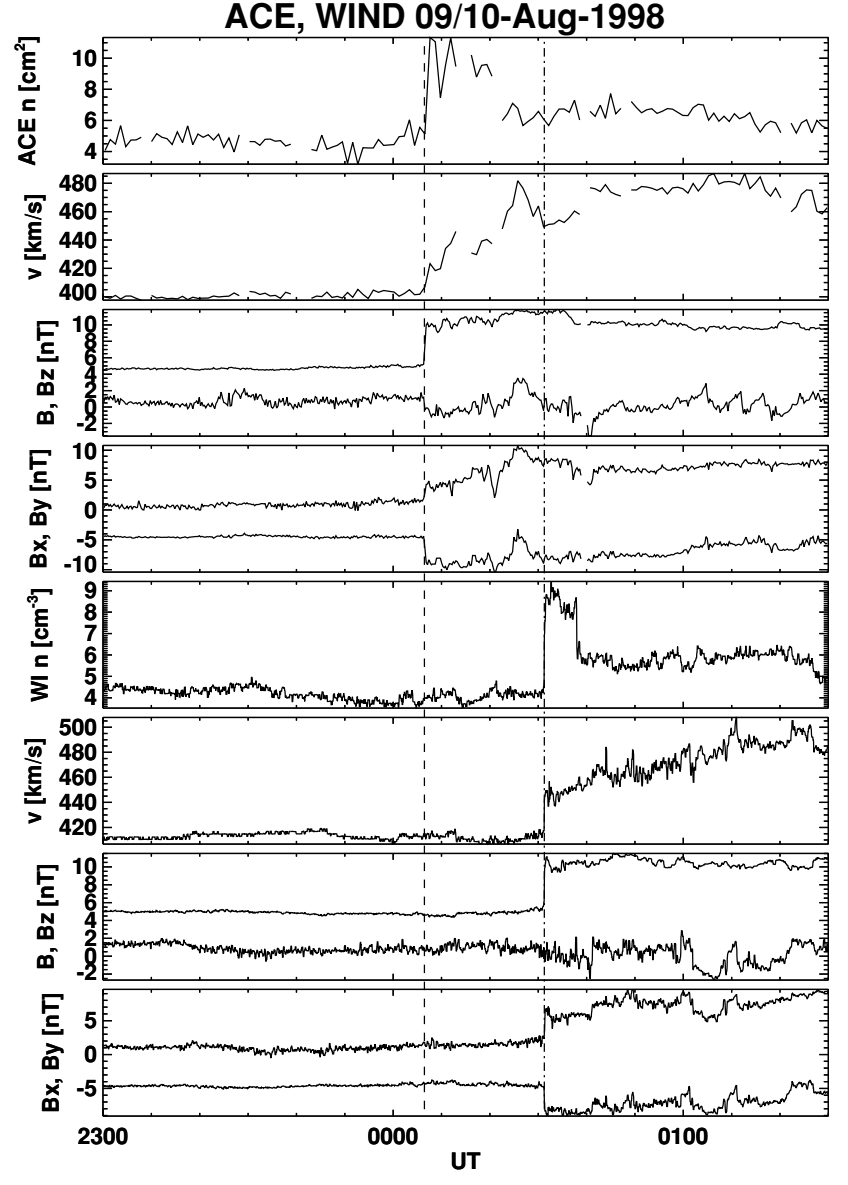

Fig. 1. Fast forward shock observed on August 10, 1998 by ACE and Wind upstream of the bow shock (thin dashed and dashed-dotted lines). From the top to bottom: ACE (first four panels) and Wind (second four panels) densities, velocities, and IMF strengths and all components.

shown in Fig. 3. The ion flux is computed as a product of the proton speed and density for all spacecraft except Interball-1 and it cover the energy range of several keV (up to $\sim 25 \mathrm{keV}$ ) depending on a particular spacecraft. Interball1 was equipped with a special set-up of for ion flux measurements without any selection of energies. Since the contribution of high-energy ions to the total ion flux is small, we assume that the different energy ranges cannot spoil the results of our qualitative study.

The originally very steep IP shock front underwent a significant evolution. The IMP 8 observation reveals similar shock features as those observed by WIND but a strong significant modification of the shock front was observed by Geotail and no similar shock-like discontinuity was detected at the Interball-1 location. Instead, Interball-1 observed short spikes of both depicted parameters approximately at the predicted time of the IP shock arrival. These spikes are followed by a gradual rise and they reached their expected post-shock values only after 10-12 minutes.

\section{Discussion and Conclusion}

The shock front modification observed by Interball-1 cannot be probably attributed to a temporal evolution because the shock withstood unchanged for $\sim 35$ minutes from ACE to IMP 8 and the expected time lag between IMP 8 


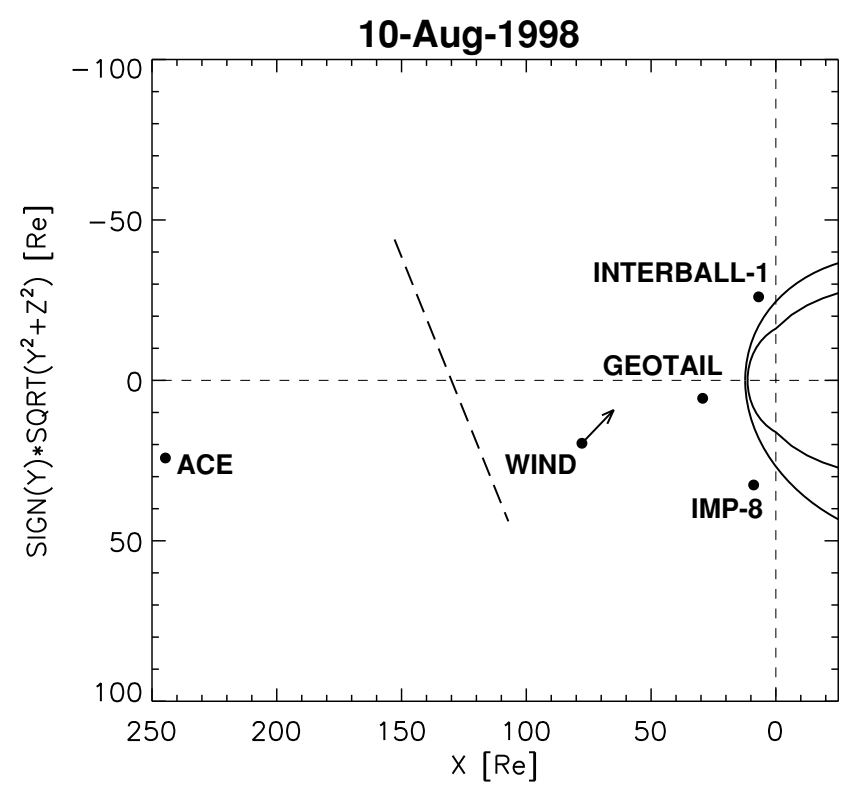

Fig. 2. Cylindrical projection of the spacecraft locations onto the equatorial plane. Jerab et al. (2005) and Petrinec and Russell (1996) models were applied to determine Earth's bow shock and magnetopause positions before the shock. Shock parameters computed from Rankine-Hugenoit relations from the Wind data are: $v_{\mathrm{sh}}=355 \mathrm{~km} / \mathrm{s}$, and $n=[-0,53 ;-0,44 ; 0,73]$ (an arrow at the Wind location). The normal and speed computed from timing of the 4 spacecraft (Wind, ACE, Geotail, IMP 8) observations (Koval et al., 2005) are: $v_{\mathrm{sh}}=319 \mathrm{~km} / \mathrm{s}$, and $n=[-0,55 ;-0,29 ; 0,77]$. The dashed line shows this shock plane.

and Interball-1 is about 7 minutes, only. On the other hand, IMP 8 was orbiting in front of the quasiperpendicular bow shock, whereas Interball-1 was moving in the quasiparallel region and Geotail was probably near the ion foreshock boundary as can be seen from the schematics in Fig. 4 (note that the ion foreshock boundary lies downstream of that of electron foreshock due to a smaller ion speed). Positions of the spacecraft as well as the magnetic field vectors are projected onto the $X Y_{\mathrm{GSE}}$ plane. The estimated location of the IP shock is shown by thin dashed lines with about twominute spacing, the heavy dashed line stands for the electron foreshock boundary after the IP shock arrival. Both upstream and downstream IMF orientations are shown by the heavy arrows.

The main foreshock feature that can influence the propagation of the IP shock is probably a presence of energetic particles because the amplitude of ULF waves was very low in our case (see Fig. 3). For this reason, we are showing the temporal profiles of several energies measured by Geotail and Interball-1 in Fig. 5. The estimated IP shock fronts are distinguished by dashed vertical lines. The Interball-1 magnetic field profile is shown for the sake of reference. The measurements of both spacecraft are, in some sense, similar. Prior the IP shock arrival, both of them observe gradually increasing particle fluxes at all energies. Their anisotropy is very low. It is shown in the last panel for Geotail as anisotropy coefficients and it can be estimated from a (lack of) spin modulation in the $F_{\mathrm{p} 2}$ panel of the Interball-1 data ( $3^{\text {th }}$ panel in Fig. 5). The anisotropy coefficients, $a 1$ and $a 2$, are amplitudes of the first and second harmonics of
WIND, IMP-8, GEOTAIL, INTERBALL 10-Aug-1998

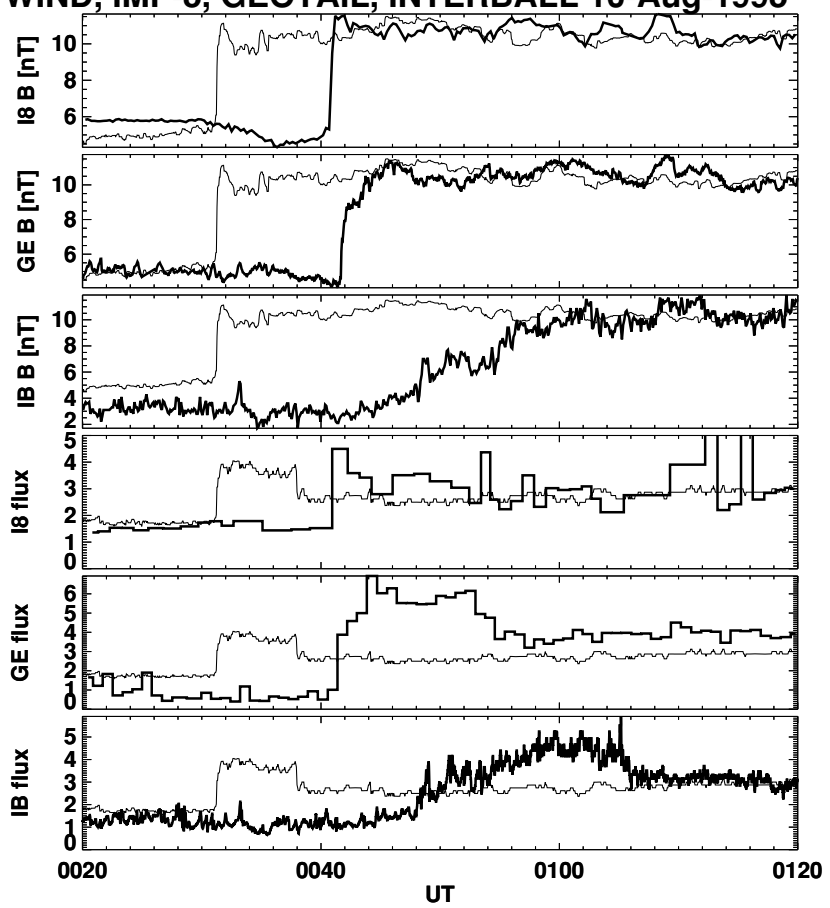

Fig. 3. IMP 8, Geotail, and Interball-1 observations of the IP shock upstream of the bow shock on August 10, 1998. From top bottom: IMF (first three panels) and ion fluxes (in units of $10^{8}\left[\mathrm{~cm}^{-2} \mathrm{~s}^{-1}\right]$ ) from IMP 8, Geotail, and Interball-1, respectively. In each panel, the IMF or ion flux from Wind (thin lines) are presented for the sake of reference.

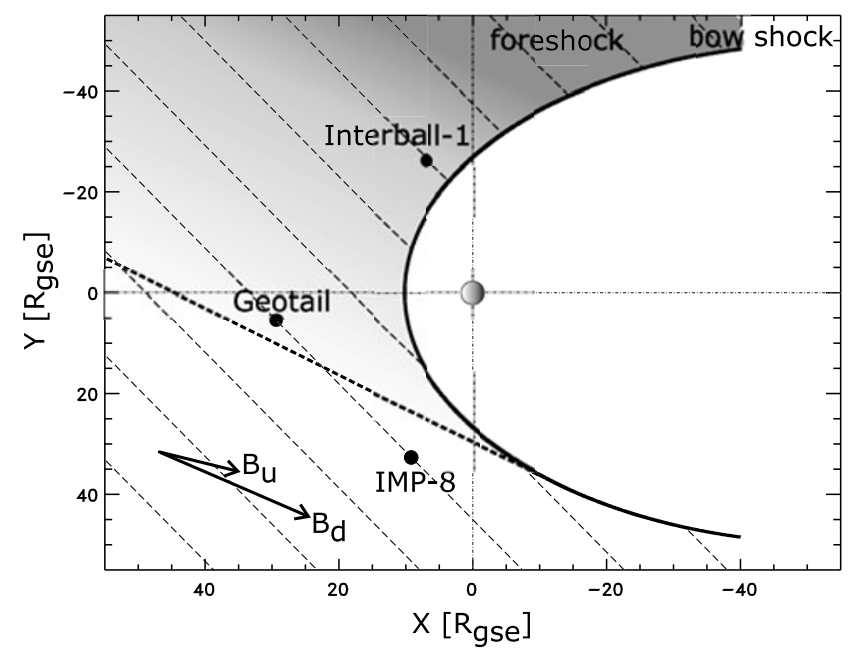

Fig. 4. The sketch of projections of spacecraft locations onto the ecliptic plane that respects their real observations in different distances from the electron foreshock boundary (heavy dashed line). The orientations of upstream $\left(B_{\mathrm{u}}\right)$ and downstream $\left(B_{\mathrm{d}}\right)$ IMF are shown by the arrows. The estimated orientation of the IP shock is depicted by thin dashed lines.

the Fourier fit to the measured ratio of paralell and perpendicular particle fluxes. The increase of the particle flux can be connected with the approaching IP shock because the flux of particles accelerated there is added to the original foreshock flux. The rise of the energetic particle flux is terminated not at but about two minutes after IP shock arrivals to both locations. After this peak, the particle flux drops down by an order of magnitude and becomes anisotropic at 


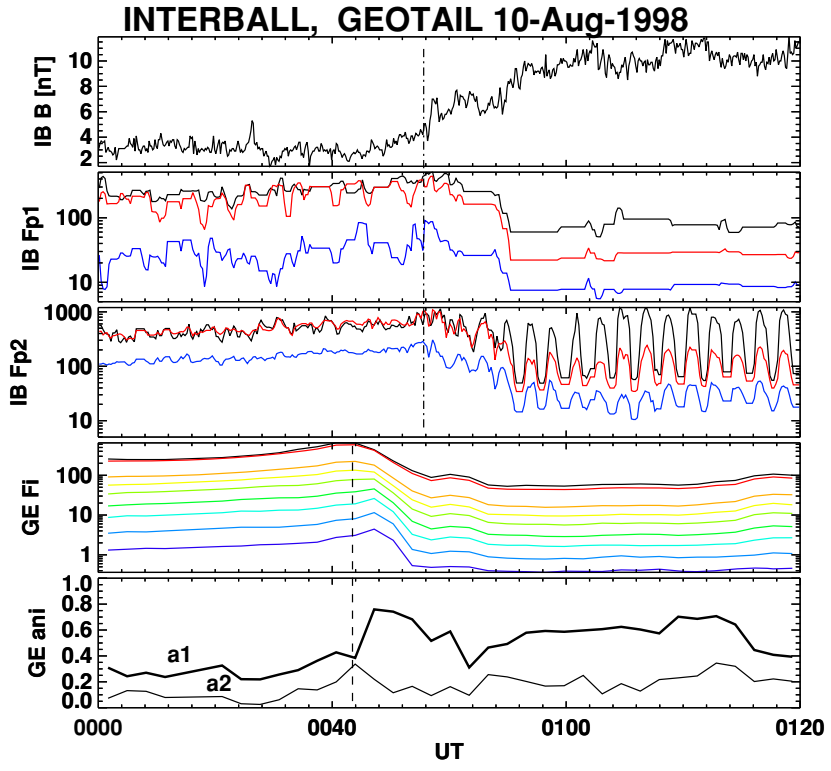

Fig. 5. The temporal profiles of energetic particles measured on Interball-1 and Geotail before and after the IP shock transition. Interball-1 (first three panels) show 3 different energies (blue line- $115 \mathrm{keV}$, red-50 keV, and black-25 keV) in two directions ( $F_{\mathrm{p} 1}$ in the sunward and $F_{\mathrm{p} 2}$ near the anti-sunward directions). Geotail panels (two bottom panels) show energetic particle fluxes ( $4^{\text {th }}$ panel from top to bottom-the energy range from 67 to $414 \mathrm{keV}$ ) and anisotropy coefficients ( $a 1$ and $a 2$ in the $5^{\text {th }}$ panel).

the Geotail location. This decrease is probably connected with the Geotail exit from the ion foreshock because the IP shock (and the downstream IMF) approached the bow shock about 2 minutes after it passed Geotail (see Fig. 4). The drop of the energetic particle flux is associated with a new rise of the magnetic field toward its post-shock equilibrium value (compare Figs. 3 and 5).

The behavior of the energetic particles at the Interball-1 location is more peculiar because their flux remains nearly isotropic for about 6 minutes after the IP shock arrival and then it jumps down and a strong anisotropy appears. An analysis of pitch angles revealed that the flux at about $90^{\circ}$ remains nearly on a previous level and only particles with low and high pitch angles disappeared. This change is accompanied with a new rise of the magnetic field strength. In this case, the delay of the change of energetic particle properties cannot be connected with reformation of the foreshock and its source is unclear.

Finally, we have presented a short case study of the IP shock propagation through the foreshock. We have shown that the profiles of basic parameters can be substantially modified, probably due to presence of energetic particles. However, the exact mechanism of this modification requires a further study that would include a modeling of particle trajectories.

Acknowledgments. The presented work was partly supported by the Research plan MSM 0021620860 that is financed by the Ministry of Education of the Czech Republic and partly by the Czech Grant Agency under Contracts 205/07/0694, and 205/06/0875. Their supports are greatly acknowledged. The authors would like to thank to teams of all spacecraft involved in this study.

\section{References}

Dryer, M., Bow shock and its interaction with interplanetary shocks, Radio Sci., 8, 893-901, 1973.

Echer, E., W. D. Gonzalez, and M. V. Alves, On the geomagnetic effects of solar wind interplanetary magnetic structures, Space Weather, 4, S06001, doi:10.1029/2005SW000200, 2006.

Fry, C. D., M. Dryer, Z. Smith, W. Sun, C. S. Deehr, and S.I. Akasofu, Forecasting solar wind structures and shock arrival times using an ensemble of models, J. Geophys. Res., 108, A1070, doi:10.1029/2002JA009474, 2003.

Gonzalez, W. D., B. T. Tsurutani, and A. L. C. De Gonzalez, Interplanetary origin of geomagnetic storms, Space Sci. Rev., 88, 529-562, 1999.

Grib, S. A., Interaction of non-perpendicular/parallel solar wind shock waves with the Earth's magnetosphere, Space Sci. Rev., 32, 43-48, 1982.

Grib, S. A., B. E. Brunelli, M. Dryer, and W.-W. Shen, Interaction of interplanetary shock waves with the bow shock-magnetopause system, J. Geophys. Res., 84, 5907-5921, 1979.

Jerab, M., Z. Nemecek, J. Safrankova, K. Jelinek, and J. Merka, A study of bow shock locations, Planet. Space Sci., 53, 85-94, 2005.

Koval, A., J. Safrankova, Z. Nemecek, and L. Prech, Deformation of interplanetary shock fronts in the magnetosheath, Geophys. Res. Lett., 32, doi:10.1029/2005GL023009, 2005.

Koval, A., J. Safrankova, Z. Nemecek, A. A. Samsonov, L. Prech, J. D. Richardson, and M. Hayosh, Interplanetary shock in the magnetosheath: Comparison of experimental data with MHD modeling, Geophys. Res. Lett., 33, doi:10.1029/2006GL025707, 2006.

Le, G., C. T. Russell, and J. G. Luhmann, Polar magnetic observations of the low-altitude magnetosphere during the January 1997 coronal mass ejection/magnetic cloud event, Geophys. Res. Lett., 25, 25332536, 1998.

McKenna-Lawlor, S. M. P., M. Dryer, M. D. Kartalev, Z. Smith, C. D. Fry, W. Sun, C. S. Deehr, K. Kecskemety, and K. Kudela, Near real-time predictions of the arrival at Earth of flare-related shocks during Solar Cycle 23, J. Geophys. Res., 111, A11103, doi:10.1029/2005JA011162, 2006.

Petrinec, S. M. and C. T. Russell, Near-Earth magnetopause shape and size as determined from the magnetopause flaring angle, J. Geophys. Res., 101, 137-152, 1996.

Russell, C. T., J. T. Gosling, R. D. Zwickl, and E. J. Smith, Multiple spacecraft observations of interplanetary shocks: ISEE three-dimensional plasma measurements, J. Geophys. Res., 88, 9941-9947, 1983.

Russell, C. T., et al., The interplanetary shock of September 24, 1998: Arrival at Earth, J. Geophys. Res., 105, 25,143-25,154, 2000.

Safrankova, J., Z. Nemecek, L. Prech, G. Zastenker, N. Nikolaeva, M. Nozdrachev, A. Skalsky, K. Paularena, and T. Mukai, January 10-11, 1997 magnetic cloud: Multipoint measurements, Geophys. Res. Lett., 25, 2549-2552, 1998.

Samsonov, A. A., Z. Nemecek, and J. Safrankova, Numerical MHD modeling of propagation of interplanetary shock through the magnetosheath, J. Geophys. Res., 111, A08210, doi:10.1029/2005JA011537, 2006.

Spreiter, J. R. and S. S. Stahara, Gasdynamic and magnetohydrodynamic modeling of the magnetosheath-a tutorial, Adv. Space Res., 14(7), 519, 1994.

Thomsen, M. F., Multispacecraft observations of collisionless shocks, Adv. Space Res., 8(9), 157, 1988.

Tsurutani, B. T. and W. D. Gonzalez, The interplanetary causes of magnetic storms: a review, in Magnetic storms, edited by B. T. Tsurutani, W. D. Gonzalez, Y. Kamide, and J. K. Arballo, AGU Monograph 98, Washington, D.C., pp. 77-90, 1997.

Vennerstroem, S., Interplanetary sources of magnetic storms: A statistical study, J. Geophys. Res., 106, 29,175-29,184, 2001.

Vinas, A. F. and J. D. Scudder, Fast and optimal solution to the RankineHugoniot problem, J. Geophys. Res., 91, 39-58, 1986.

Yan, M. and L. C. Lee, Interaction of interplanetary shocks and rotational discontinuities with the Earth's bow shock, J. Geophys. Res., 101, 48354848, 1996.

Zhuang, H. C., C. T. Russell, E. J. Smith, and J. T. Gosling, Threedimensional interaction of interplanetary shock waves with the bow shock and magnetopause: A comparison of theory with ISEE observations, J. Geophys. Res., 86, 5590-5600, 1981.

L. Přech (e-mail: lubomir.prech@mff.cuni.cz), Z. Němeček, and J. Šafránková 